\title{
Plasmon-Driven Motion of an Individual Molecule
}

\author{
Tzu-Chao Hung, Brian Kiraly, Julian H. Strik, Alexander A. Khajetoorians, and Daniel Wegner* \\ Cite This: Nano Lett. 2021, 21, 5006-5012 \\ Read Online
}

ACCESS

Ш Metrics \& More

Article Recommendations

Supporting Information

ABSTRACT: We demonstrate that nanocavity plasmons generated a few nanometers away from a molecule can induce molecular motion. For this, we study the well-known rapid shuttling motion of zinc phthalocyanine molecules adsorbed on ultrathin $\mathrm{NaCl}$ films by combining scanning tunneling microscopy (STM) and spectroscopy (STS) with STM-induced light emission. Comparing spatially resolved single-molecule luminescence spectra from molecules anchored to a step edge with isolated molecules adsorbed on the free surface, we found that the azimuthal modulation of the Lamb shift is diminished in case of the latter. This is evidence that the rapid shuttling motion is remotely

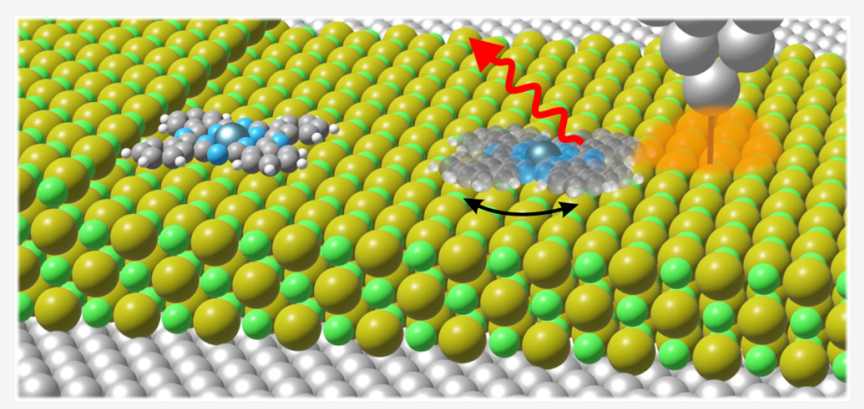
induced by plasmon-molecule coupling. Plasmon-induced molecular motion may open an interesting playground to bridge the nanoscopic and mesoscopic worlds by combining molecular machines with nanoplasmonics to control directed motion of single molecules without the need for local probes.

KEYWORDS: scanning tunneling microscopy (STM), STM-induced light emission, single-molecule spectroscopy, plasmon-molecule coupling, molecular manipulation, zinc phthalocyanine

$\mathrm{C}$ ontrolled activation of molecular motion and conformational changes is vital for creating molecular devices, motors, and machines. ${ }^{1,2}$ To this end, molecular motion can be induced by a variety of physical and chemical stimuli, depending on the desired functionality. ${ }^{3}$ While nonlocal stimuli, such as an external light source, can address many molecular machines simultaneously, ${ }^{4-6}$ it is ultimately desirable to create molecular devices where molecules can be addressed individually. ${ }^{7-11}$ Controlled on-surface manipulation of single atoms and molecules has been successfully demonstrated using a scanning tunneling microscope (STM), typically via electronic or inelastic (vibrational or vibronic) excitation mechanisms. ${ }^{8-10,12-18}$ Within the tip-sample junction, nanocavity plasmons can also be excited, ${ }^{19}$ as seen in STM-induced light emission (STM-LE). ${ }^{20-23}$ While plasmon-induced chemical reactions have been reported, ${ }^{24}$ to date there has been no demonstration that local nanocavity plasmon-molecule coupling can also be utilized to induce single-molecule motion.

Phthalocyanine $(\mathrm{Pc})$ molecules and their metal complexes on ultrathin $\mathrm{NaCl}$ films serve as a model platform to fundamentally investigate optoelectronic phenomena and local interactions on the single-molecule scale using STM$\mathrm{LE}$, including recent discoveries of single-photon emission, ${ }^{25}$ up-conversion electroluminescence, ${ }^{26}$ resonance energy transfer, ${ }^{27}$ coherent dipole coupling and superradiance, ${ }^{28,29}$ coherent plasmon-exciton interactions, ${ }^{30,31}$ transient charging and electrofluorochromism, ${ }^{32,33}$ vibronic spectroscopy, $^{30,32,34,35}$ tautomerization tracking, ${ }^{35}$ and doublet emis- sion. ${ }^{36}$ Zinc phthalocyanine $(\mathrm{ZnPc})$ is particularly interesting to investigate the possibility of plasmon-induced molecular motion. On the one hand, a rapid shuttling motion between two equivalent adsorption configurations can be induced. ${ }^{27-29,32,34,37-43}$ On the other hand, nanocavity plasmons in the tip-sample junction can remotely couple to the molecule, creating an exciton and eventually leading to light emission. $^{27,30-32,35,44}$ This coherent coupling leads to a Lamb shift of the optical transition, which was found to vary with azimuthal angle of the STM tip along a circumferential path around the molecule, reflecting the $\mathrm{ZnPc}$ symmetry. ${ }^{31,45}$ However, thus far there has been no indication that this remote plasmon-molecule coupling may also induce the rapid shuttling motion.

Here, we show that plasmons generated in a nanocavity can induce molecular motion. We quantify this effect, using a combination of STM, scanning tunneling spectroscopy (STS), and STM-LE. We compare two types of electronically equivalent $\mathrm{ZnPc}$ molecules, as seen by STS, one being isolated species which can exhibit rapid shuttling and the other being molecules that are anchored to $\mathrm{NaCl}$ step edges in order to suppress the shuttling. By comparing the azimuthal depend-

Received: February 24, 2021

Revised: $\quad$ May 19, 2021

Published: June 1, 2021 
ence of STM-LE spectra of these two ZnPc types, we observe a remarkable difference-that the azimuthal modulation of the Lamb shift for anchored molecules is much larger than for isolated ones. Our findings can be understood in a straightforward model, revealing that rapid shuttling of isolated molecules is induced not only when directly tunneling into the $\mathrm{ZnPc}$ but even at remote distances a few nanometers away, where only plasmons couple to the molecule. The fact that nanocavity plasmons can induce single-molecule motion opens up an unexplored route toward inducing and controlling molecular motion by using prepatterned nanoplasmonic structures.

Figure 1(b) shows a constant-current STM image of a typical isolated $\mathrm{ZnPc}$ adsorbed on a free terrace of $3 \mathrm{ML}$

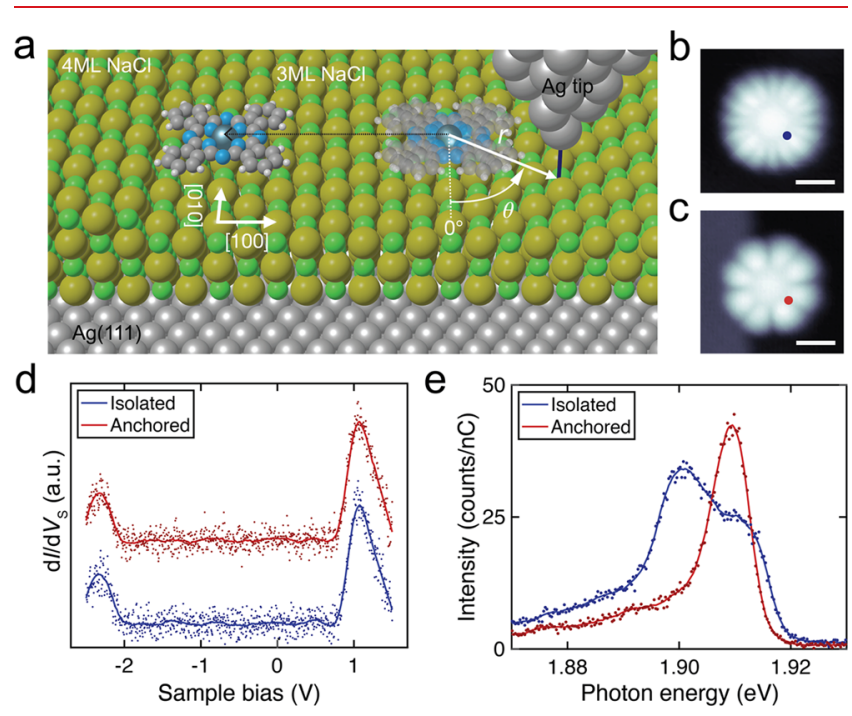

Figure 1. Isolated vs anchored $\mathrm{ZnPc}$ on $3 \mathrm{ML} \mathrm{NaCl} / \mathrm{Ag}(111)$. (a) Schematics of STM-LE and manipulation experiments. The structural model illustrates the $\pm 11^{\circ}$ shuttling between two adsorption orientations for isolated $\mathrm{ZnPc}$ (right), which is suppressed for anchored $\mathrm{ZnPc}$ (left). The lateral molecule-tip distance $r$ and azimuthal angle $\theta$ are defined from the $\mathrm{ZnPc}$ center, with $\theta=0^{\circ}$ defined downward along the $\mathrm{NaCl}[0 \overline{10}]$ direction as displayed. (b) Constant-current STM image of an isolated $\mathrm{ZnPc}$ before manipulation, and (c) anchored $\mathrm{ZnPc}$ after moving it to a $4 \mathrm{ML} \mathrm{NaCl}$ step edge $\left(V_{\mathrm{S}}=-2.5 \mathrm{~V}, I=5.5 \mathrm{pA}\right.$, scale bars $\left.=1 \mathrm{~nm}\right)$. (d) STS of the isolated and anchored $\mathrm{ZnPc}$. (e) STM-LE spectra of the isolated and the anchored $\mathrm{ZnPc}\left(V_{\mathrm{S}}=-2.5 \mathrm{~V}, I=100 \mathrm{pA}\right.$, acquisition time $t=180$ s) measured at the positions marked in (b) and (c). Solid lines in (d) and (e) are 80-point and 20-point Savitzky-Golay filtered curves of the raw data, respectively.

$\mathrm{NaCl} / \mathrm{Ag}(111)$ (see Supporting Information Section S1 for experimental details) when resonantly tunneling out of the highest occupied molecular orbital (HOMO). The HOMO hosts eight lobes (two at each of the four ligand phenyl groups) (see Supporting Information Section S2). ${ }^{29}$ Yet, in the STM image 16 lobes can be observed, due to a superposition of two bistable orientations on the same adsorption site. This apparent superposition results from the rapid shuttling of the molecule about the $\mathrm{Zn}$ atom between two bistable orientations, where the phenyl groups are oriented either $+11^{\circ}$ or $-11^{\circ}$ with respect to the $\mathrm{NaCl}\langle 110\rangle$ crystallographic directions ${ }^{37}$ (see Figure 1(a)). It is known that rapid shuttling occurs when electrons resonantly tunnel either out of the HOMO or into the lowest unoccupied molecular orbital
(LUMO). ${ }^{25,28,29,31,32,34,37}$ For a wide range of voltages within the HOMO-LUMO gap, the molecule can be stabilized in one of the two aforementioned orientations. ${ }^{37,39,40}$ However, the shuttling motion can also be induced for certain in-gap voltages, ${ }^{37,39}$ which raises questions on the mechanism that activates this motion (see also Supporting Information Section S6).

In order to quantify the role of the nanocavity plasmons on this rapid shuttling, we first create a reference molecule that does not exhibit shuttling motion. While defects in the $\mathrm{NaCl}$ substrate can pin molecules and suppress the shuttling motion, ${ }^{37}$ we found that $\mathrm{ZnPc}$ pinned to defects can exhibit strongly modified electronic and/or optical properties (see Supporting Information Section S3). Therefore, we used STM manipulation $^{29}$ to move an isolated molecule to the edge of a $\mathrm{NaCl}$ step, between 3 and $4 \mathrm{ML}$. We only used binding sites free of local adsorbates or apparent defects, oriented along the $\langle 100\rangle$ direction. Figure 1(c) presents the constant-current STM image of the same $\mathrm{ZnPc}$ shown in (b) after the aforementioned manipulation procedure. The adsorption site is identical to that of isolated molecules, with the $\mathrm{Zn}$ atom located above a $\mathrm{Cl}$ atom. However, at the imaging voltage in which the isolated molecule exhibits shuttling motion, only eight lobes, correlated with the HOMO, were observed for molecules relocated to the step edge. In comparison with the isolated molecule, the relocated molecule was stabilized such that the phenyl groups almost align along the $\mathrm{NaCl}\langle 110\rangle$ directions, as shown in Figure 1(a). In the following, we refer to this molecule as anchored $\mathrm{ZnPc}$.

Before comparing the optical properties of isolated and anchored $\mathrm{ZnPc}$, we first demonstrate that the local density of states (LDOS) is identical in both cases. In Figure 1(d), STS spectra are taken for both molecules at locations marked in (b) and (c). In both cases, the HOMO (i.e., the positive ion resonance $^{46}$ ) is found at $-2.33 \mathrm{eV}$ below the Fermi energy $\left(E_{\mathrm{F}}\right)$, and the LUMO (negative ion resonance) is located at $1.05 \mathrm{eV}$ above $E_{\mathrm{F}}$. Likewise, the spatial maps of the HOMO and LUMO do not illustrate any modifications in the spatial LDOS distribution compared to the isolated molecule apart from the blurring due to the rapid shuttling of the latter (see Supporting Information Section S2). We note that it is important to ensure a clean surface and step edge, as even defects a few nanometers away from the molecule can alter the measured $\mathrm{d} I / \mathrm{d} V_{\mathrm{S}}$ of the molecule (see Supporting Information Section S3). Previous work indicates that a different adsorption site would be accompanied by a different STS spectrum, ${ }^{36}$ which is further evidence that the molecular adsorption site in both cases is a $\mathrm{Cl}$ site. Hence, we conclude that the relocated molecule is not chemisorbed to the step edge, but weakly physisorbed. We speculate that the anchoring of the molecule most likely occurs due to additional van der Waals interactions, which suppress the shuttling motion without altering the internal structural and electronic properties of the molecule. Therefore, in the subsequent discussion, we treat the isolated and anchored molecule as electronically and chemically equivalent.

We now compare the STM current-induced fluorescence of isolated versus anchored $\mathrm{ZnPc}$, first focusing on the overall spectral features. As previously reported, the STM-LE spectrum on top of the isolated $\mathrm{ZnPc}$ (blue curve in Figure $1(\mathrm{e})$ ) illustrates two features, i.e., a broad peak at $1.902 \mathrm{eV}$ and a shoulder at $1.911 \mathrm{eV}^{25,28,29,31,32,34,37}$ While the former has been interpreted as the $\mathrm{Q}(0,0)$ singlet transition fluorescence 

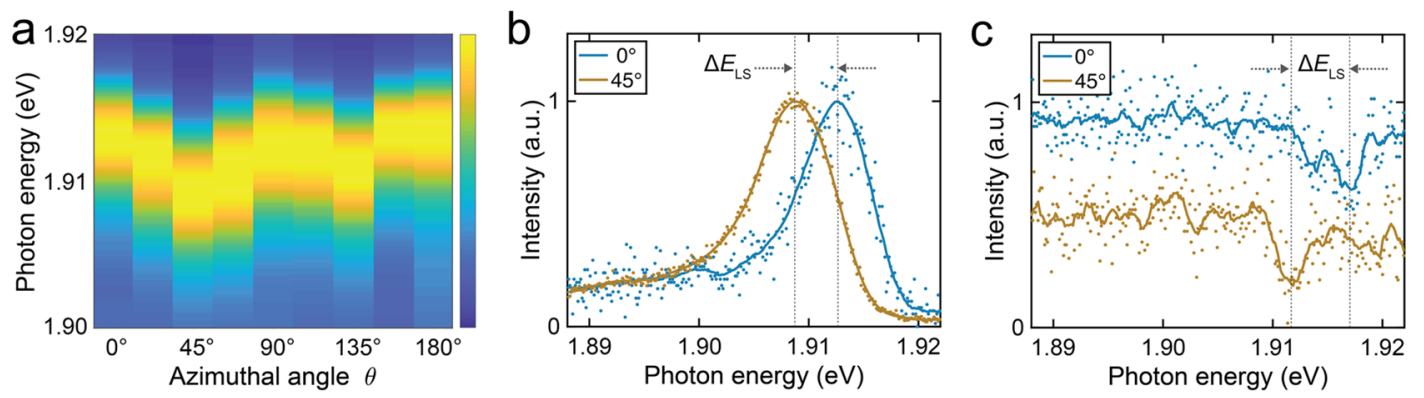

Figure 2. Lamb shift modulation (LSM) of anchored ZnPc. (a) Color plot of normalized STM-LE spectra showing periodic modulation of the Lamb shift. (b) Two typical Fano-peak STM-LE spectra taken on top of anchored $\mathrm{ZnPc}(r=0.83 \mathrm{~nm})$ for $\theta=0^{\circ}$ and $45^{\circ}$, with $\Delta E_{\mathrm{LS}} \approx 3.9 \pm 0.3$ $\mathrm{meV}$ (taken in constant-height mode with the feedback loop opened at the $\mathrm{ZnPc}$ center, $V_{\mathrm{S}}=-2.5 \mathrm{~V}, I=500 \mathrm{pA}, t=120 \mathrm{~s}$ ). (c) Two typical Fanodip STM-LE spectra taken next to anchored $\mathrm{ZnPc}(r=1.81 \mathrm{~nm})$ at $\theta=0^{\circ}$ and $45^{\circ}$, with $\Delta E_{\mathrm{LS}} \approx 5.2 \pm 0.5 \mathrm{meV}$ (constant-current mode $V_{\mathrm{S}}=-2.5$ $\mathrm{V}, I=200 \mathrm{pA}, t=60 \mathrm{~s}$ ). Solid lines in (b) and (c) are 50-point and 20-point Savitzky-Golay filtered curves of the raw data, respectively.

peak, it has been suggested that the latter is related to the shuttling motion of the molecule, as it is not observed within aggregated molecule chains. ${ }^{28,29,34}$ In comparison, the STMLE spectrum on top of the anchored molecule (red curve in Figure 1(e)) shows a single peak located at a different energy $(1.910 \mathrm{eV})$ with a much narrower width and no shoulder feature. We attribute this peak to the $Q(0,0)$ transition. Its blueshift with respect to the isolated molecule can be explained by the different dielectric environment defined by the step edge and/or by additional van der Waals interactions, leading to a modified exciton binding energy. ${ }^{29}$ Interestingly, the asymmetric spectral shape reflects a Fano line shape, indicating the presence of two coherent excitation channels. The two potential excitation channels discussed in the literature are $(i)$ excitation resulting from direct tunneling into the molecule, i.e., electron and hole injection into frontier orbitals, leading to the formation of an exciton,,$^{29,37}$ and (ii) inelastic excitation resulting from tunneling between tip and substrate, exciting nanocavity plasmons and permitting a plasmon-induced energy transfer and exciton formation in the molecule. ${ }^{32,34}$ While previous studies concluded that either $(i)$ or (ii) alone is responsible for the observed STM-LE spectrum for the isolated molecule, our finding clearly shows that both excitation mechanisms are activated and couple coherently, resulting in the observed Fano resonance. ${ }^{31,44}$ This conclusion can be made due to the sharper spectral feature and clear line shape presented for the anchored molecule. Therefore, it is important to elucidate a possible impact of nanocavity plasmons on inducing the shuttling motion observed for isolated molecules.

In order to quantify the role of nanocavity plasmons in molecule-plasmon coupling, we focus on the Lamb shift. The Lamb shift is a redshift in the molecular optical response due to a coherent coupling of the discrete molecular emitter state with the continuum of states of the nanocavity plasmons created in the tip-sample junction. ${ }^{31,47-49}$ We employed it as a highly sensitive remote probe for the molecular orientation of the $\mathrm{ZnPc}$ with respect to the $\mathrm{NaCl}$ substrate. This is possible because the Lamb shift was recently found to be modulated along a circle around the molecule with a $\pi / 2$ periodicity in the azimuthal direction. ${ }^{31}$ This Lamb shift modulation (LSM) was attributed to the $\mathrm{D}_{4 \mathrm{~h}}$ symmetry of the $\mathrm{ZnPc}$ with its two orthogonal transition dipole moments along the main molecular axes, leading to an anisotropic coupling of the molecule with the nanocavity plasmons. However, a rapid shuttling would diminish the detected effective LSM, as a superposition of two phase-shifted LSMs from the bistable orientations of the molecule would be observed. So far, STMLE-based Lamb shift measurements have only been performed remotely, with the tip a few nanometers away from the molecule, ${ }^{31}$ but no azimuthal modulation of the Lamb shift has been reported when directly tunneling into the isolated molecule, presumably because it may be invisible due to the rapid shuttling and the ensuing spectral broadening.

To acquire a reference of the LSM for $\mathrm{ZnPc}$ both on and off the molecule, we used the STM-LE spectra from an anchored ZnPc. Figure 2(a) presents a series of false-color plotted STM$\mathrm{LE}$ spectra of the anchored $\mathrm{ZnPc}$ at a radial distance from the molecular center (i.e., the $\mathrm{Zn}$ atom) of $r=0.83 \mathrm{~nm}$ (i.e., on the Pc ligand) at different azimuthal angles $\theta$. Here, $\theta=0^{\circ}$ is defined along the crystallographic $\mathrm{NaCl}[0 \overline{1} 0]$ direction as shown in Figure 1(a). To maintain comparable nanocavity plasmons for each $\theta$, the STM-LE spectra were recorded in constant-height mode. The resulting series of STM-LE spectra revealed that the emission peak position approximately oscillates with a $\pi / 2$ periodicity owing to the azimuthal modulation of the Lamb shift. The nonequal energy level shift at $45^{\circ}$ and $135^{\circ}$ can be explained by an asymmetric tip shape (see Supporting Information Section S4 for more details). In Figure 2(b), STM-LE spectra at $\theta=0^{\circ}$ (blue curve) and at $\theta=$ $45^{\circ}$ (orange) are shown. The Lamb shift is largest at $\theta=45^{\circ}$, leading to a stronger redshift with increased moleculeplasmon coupling compared to the STM-LE spectrum at $\theta=$ $0^{\circ}$. A fit of the STM-LE spectra with a Fano function (see Supporting Information Section S5) reveals comparable widths and $q$ factors, with a LSM of $\Delta E_{\mathrm{LS}}=E_{0}\left(0^{\circ}\right)-E_{0}\left(45^{\circ}\right)=3.9 \pm$ $0.3 \mathrm{meV}$. In comparison, Zhang et al. reported a much smaller LSM of $\Delta E_{\mathrm{LS}} \approx 2 \mathrm{meV}$ for isolated molecules. ${ }^{31}$ The latter was measured not on but next to the molecule $(r \geq 1.6 \mathrm{~nm})$, and the Lamb shift is known to exhibit a lateral tip-molecule distance dependence, ${ }^{31}$ which may explain the larger LSM measured here at $r=0.83 \mathrm{~nm}$. To test this, we also performed experiments at various distances. Figure 2(c) shows two STMLE spectra obtained at $r=1.81 \mathrm{~nm}$ (i.e., the tip was positioned next to the molecule) at an angle of $0^{\circ}$ (blue) and $45^{\circ}$ (orange), respectively. At this distance, direct tunneling into the molecule is not possible (hence, the excitation channel $(i)$ can be completely ruled out) and the molecular resonance is only observed indirectly via plasmon-exciton coupling, leading to a faint Fano dip in the plasmon resonance STM-LE spectrum. $^{31,44}$ Irrespective of the small signal-to-noise ratio, these remote STM-LE spectra also display a clear LSM, which we quantified as $\Delta E_{\mathrm{LS}} \approx 5.2 \pm 0.5 \mathrm{meV}$. Additional STM-LE 

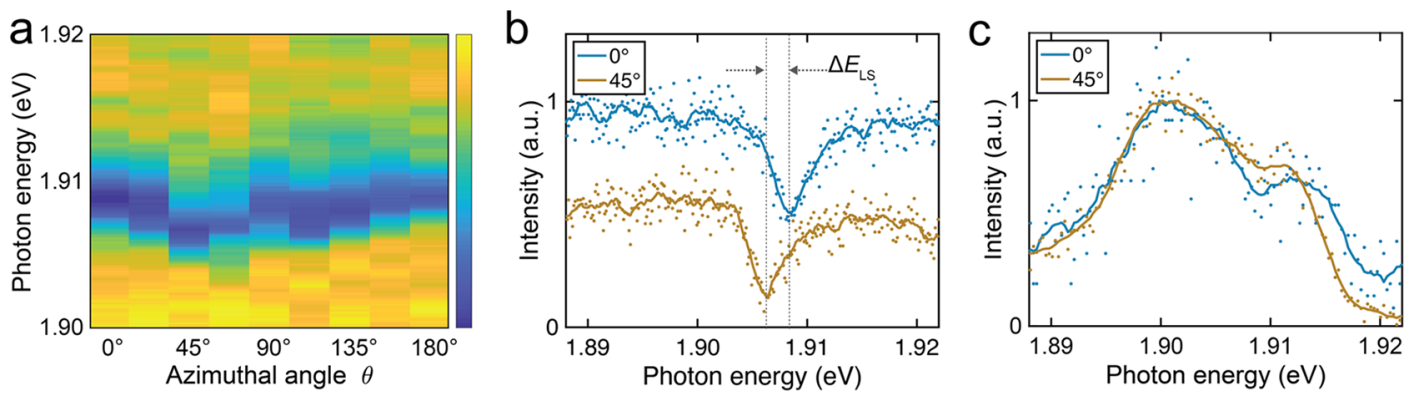

Figure 3. LSM of isolated ZnPc. (a) Color plot of normalized STM-LE spectra showing periodic modulation of the Lamb shift. (b) Two typical Fano-dip STM-LE spectra taken next to isolated $\mathrm{ZnPc}(r=1.84 \mathrm{~nm})$ at $\theta=0^{\circ}$ and $45^{\circ}$, with $\Delta E_{\mathrm{LS}} \approx 2.5 \pm 0.3$ meV (constant-current mode, $V_{\mathrm{S}}=$ $-2.5 \mathrm{~V}, I=200 \mathrm{pA}, t=120 \mathrm{~s})$. (c) Two STM-LE spectra obtained on top of isolated ZnPc $(r=0.83 \mathrm{~nm})$ at $\theta=0^{\circ}$ and $45^{\circ}$, with no assignable LSM (constant-height mode, feedback opened at ZnPc center, $V_{\mathrm{S}}=-2.5 \mathrm{~V}, I=100 \mathrm{pA}, t=60 \mathrm{~s}$ ). Solid lines in (b) and (c) are 200-point and 20point Savitzky-Golay filtered curves of the raw data, respectively.
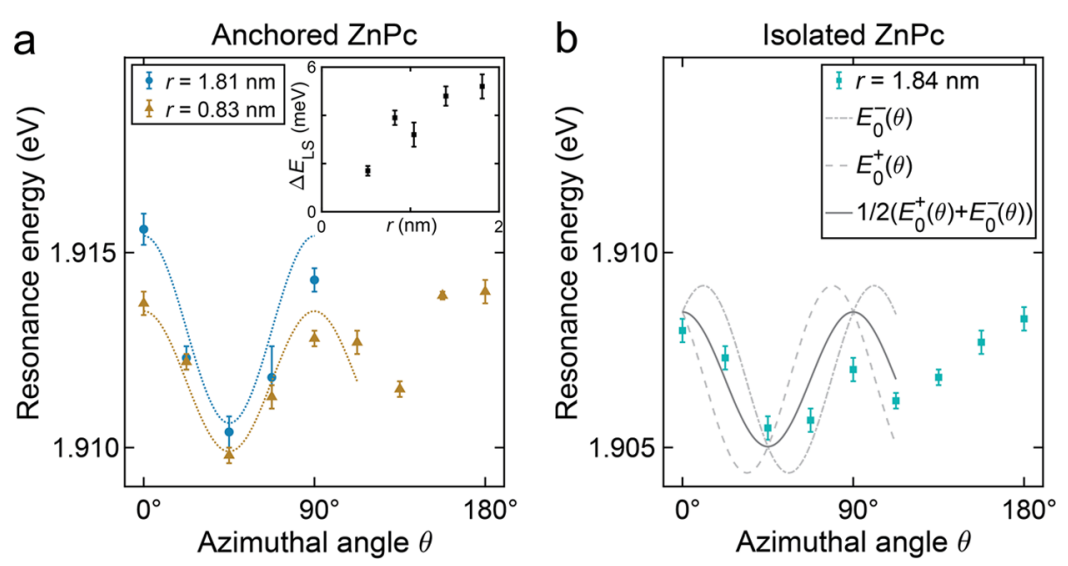

Figure 4. Analysis of the LSM for anchored vs isolated ZnPc. (a) Azimuthal angle dependence of the $\mathrm{Q}(0,0)$ resonance for anchored $\mathrm{ZnPc}$ at $r=$ $0.83 \mathrm{~nm}$ (orange triangles) and $r=1.81 \mathrm{~nm}$ (blue circles). A cosine fit yields peak-to-peak amplitudes $2 A=\Delta E_{\mathrm{LS}}$. The inset shows a summary of the radius-dependent $\Delta E_{\mathrm{LS}}$ for various $r$. (b) Azimuthal angle dependence of the $\mathrm{Q}(0,0)$ resonance for isolated $\mathrm{ZnPc}$ at $r=1.84 \mathrm{~nm}$ (light-blue squares). The gray dashed lines are model curves based on results from (a) for assumed azimuthal angles of $-11^{\circ}$ and $+11^{\circ}$, respectively. Their superposition (solid gray line) reflects the expected LSM in the case of rapid shuttling. The model agrees well with the raw data.

series at various radial distances confirm this observation (cf. inset of Figure 4(a) and Supporting Information Figure S8).

In order to rule out that the enhanced LSM of the anchored molecule originates from any other artifacts, we performed the same angular-dependent measurements next to the isolated molecule on $3 \mathrm{ML} \mathrm{NaCl} / \mathrm{Ag}(111)$ with the identical tip used in Figure 2 as well as the same stabilization parameters. Figure 3(a) shows two STM-LE spectra for a radial distance of $r=$ $1.84 \mathrm{~nm}$ at $\theta=0^{\circ}$ and $45^{\circ}$, respectively (where $\theta$ is defined as before, see Figure $1(\mathrm{a}))$. Again, a $\pi / 2$ periodicity can be observed as was seen in Figure 2. Likewise, the unequal energy shift of $45^{\circ}$ and $135^{\circ}$ presumably originated from the same asymmetric tip. Fitting the $0^{\circ}$ and $45^{\circ}$ STM-LE spectra with Fano profiles, we found $\Delta E_{\mathrm{LS}} \approx 2.5 \pm 0.3 \mathrm{meV}$. This is consistent with the value previously reported ${ }^{31}$ and confirms that the LSM of isolated $\mathrm{ZnPc}$ is only about half of the value seen for the anchored molecule. We also tested whether the LSM can be observed for isolated molecules when the tip is positioned above the molecule. Figure 3(c) shows two STMLE spectra at a radial distance of $r=0.83 \mathrm{~nm}$, which reproduces the STM-LE spectrum of isolated $\mathrm{ZnPc}$ in Figure $1(\mathrm{e})$. However, due to the spectral broadening of the $\mathrm{Q}(0,0)$ resonance peak and the additional shoulder feature, no clear difference between the STM-LE spectra could be observed.

For a statistically better quantification of the LSM, we determined the $\mathrm{Q}(0,0)$ resonance energies at various $\theta$ and $r$.
Figure 4(a) summarizes data for the anchored molecule, both above the $\mathrm{ZnPc}$ ligand ( $r=0.83 \mathrm{~nm}$, orange triangles) and for the remote measurement ( $r=1.81 \mathrm{~nm}$, blue circles). The LSM remains comparably large. All data sets can be phenomenologically described as a periodic pattern using the function ${ }^{31} E_{0}$ $(\theta)=A \cos 4 \theta+B$, where a statistical value of the LSM is given by $2 A=\Delta E_{\mathrm{LS}}$ and the offset $B$ is the angle-averaged resonance energy. Fitting the data, we found $2 A(r=0.83 \mathrm{~nm})=3.6 \pm 0.4$ $\mathrm{meV}$ and $2 A(r=1.81 \mathrm{~nm})=4.8 \pm 0.6 \mathrm{meV}$. The radial distance dependence (inset of Figure $4(\mathrm{a})$ ) shows that $\Delta E_{\mathrm{LS}}$ decreases when the tip approaches the $\mathrm{ZnPc}$ center, reflecting the lateral dimensions of the nanoplasmonic cavity and the molecule; for symmetry reasons, the LSM must vanish for $r=$ $0 .^{31}$

As the LSM measurements of the anchored and the isolated $\mathrm{ZnPc}$ were performed under identical conditions (same tip and similar vertical and lateral tip-molecule distances used in Figure 2(c) and 3(b)), a quantitative comparison is possible. Figure 4(b) summarizes the angle-dependent remote measurements for the isolated molecule. The significantly diminished LSM can neither be attributed to a reduced coupling of the nanocavity plasmons to the $\mathrm{ZnPc}$ molecule nor to any other experimental artifact. This implies that remote measurements of isolated molecules are more invasive than previously suggested and that the plasmon-molecule coupling alone may induce shuttling of the isolated $\mathrm{ZnPc}$ molecule. We can 
model an expected LSM of isolated $\mathrm{ZnPc}$ that is rapidly shuttling during a measurement by a superposition $E_{0}(\theta)=\frac{1}{2}\left(E_{0}^{+}+E_{0}^{-}\right)+B$ of the LSMs originating from the two orientation angles, $E_{0}^{ \pm}(\theta)=A \cos \left[4\left(\theta \pm 11^{\circ}\right)\right]$. We used an amplitude $2 A=4.8 \mathrm{meV}$ that corresponds to the LSM of anchored $\mathrm{ZnPc}$ at $r=1.81 \mathrm{~nm}$. The resulting model curve is displayed as a solid black line in Figure 4(b). Despite its simplicity, the model describes the observed LSM of isolated $\mathrm{ZnPc}$ quite well. Hence, we conclude that the nanocavity plasmons generated a few nanometers away from $\mathrm{ZnPc}$ can induce the rapid shuttling motion. In order to further verify this, we repeatedly imaged isolated $\mathrm{ZnPc}$ molecules after each remote measurement, using in-gap voltages where the molecules do not shuttle (cf. Supporting Information Figure S9), and we indeed found that their orientation changed (Figure S10). We also performed voltage- and currentdependent measurements of switching dynamics in order to rule out other possible switching mechanisms, such as electric fields or ballistic transport of hot electrons (see Supporting Information Section S6).

A recent study on various metal phthalocyanines concluded that the rapid shuttling motion upon direct tunneling into the molecule involves an intermediate charged molecular state. ${ }^{40,43}$ Our results provide evidence that, in the case of remote measurements, i.e., in the absence of direct transport through the molecule, plasmon-molecule coupling induces the shuttling. It remains unclear how exactly nanocavity plasmons couple to the mechanical degrees of freedom. A recent $\mathrm{H}_{2} \mathrm{Pc}$ tautomerization study suggests that molecular motion may be activated exclusively by excitation from its electronic $S_{0}$ ground state into the $S_{1}$ excited singlet state, leading to vibronic relaxation. ${ }^{35}$ While this plasmon-exciton coupling mechanism is able to explain rapid shuttling at voltage magnitudes above the $S_{1}$ onset energy, we find that shuttling can already be activated remotely at much smaller bias (cf. Supporting Information Figure S10). Therefore, we suggest that nanocavity plasmon modes can directly couple to vibrationally excited modes within the $S_{0}$ state. A theoretical treatment of a related molecule $(\mathrm{MgPc})$ found an energy barrier between the two adsorption orientations of $9 \mathrm{meV}$, 39 which interestingly corresponds to the energy difference observed between the $\mathrm{Q}(0,0)$ transition and the shoulder in STM-LE of isolated $\mathrm{ZnPc}$ (Figure 1(e) and Figure 3(c)). Such a barrier can easily be overcome if a plasmon couples to a vibrational or hindered rotational mode. ${ }^{34}$ While we found connections between the plasmon resonance intensity and the shuttling dynamics (see Supporting Information Section S6) in support of this interpretation, the strong polarity dependence of the threshold biases calls for future studies with improved time resolution. $^{35,50}$

In summary, we studied the influence of the rapid shuttling motion on the fluorescence properties of $\mathrm{ZnPc}$ on the $\mathrm{NaCl}$ surface, by comparing the LSMs of an anchored and an isolated molecule. For the latter, we found a diminished LSM, which provides evidence that isolated molecules rapidly shuttle during remote measurements due to plasmon-molecule coupling. Our results should provide valuable input for future theoretical treatments to identify the exact mechanism that leads to molecular motion based on coupling to nanocavity plasmons. A better understanding could also offer a route toward inducing lateral or even directed motion of accordingly designed molecules on surfaces. Even more, it may provide a novel playground for controlling the motion of molecular machines such as rotors, motors, or nanocars, ${ }^{7,9,14,51}$ without the need for a local probe by utilization of nanoplasmonic structures, ${ }^{19,52}$ which can readily be made using state-of-the-art lithography techniques-an intriguing prospect toward scalable control of complex molecular machines.

\section{ASSOCIATED CONTENT}

\section{Supporting Information}

The Supporting Information is available free of charge at https://pubs.acs.org/doi/10.1021/acs.nanolett.1c00788.

Further details about the experimental setup and methods, characterization of the nanoplasmonic cavity, influence of defects on the measurements, molecular orbital mapping of $\mathrm{ZnPc}$, the fit analysis of STM-LE spectra, the verification of remote shuttling, and quantification of the switching behavior (PDF)

\section{AUTHOR INFORMATION}

\section{Corresponding Author}

Daniel Wegner - Institute for Molecules and Materials, Radboud University, 6525 AJ Nijmegen, The Netherlands; 다이.org/0000-0002-1625-2830;

Email: daniel.wegner@ru.nl

\section{Authors}

Tzu-Chao Hung - Institute for Molecules and Materials, Radboud University, 6525 AJ Nijmegen, The Netherlands

Brian Kiraly - Institute for Molecules and Materials, Radboud University, 6525 AJ Nijmegen, The Netherlands

Julian H. Strik - Institute for Molecules and Materials, Radboud University, 6525 AJ Nijmegen, The Netherlands

Alexander A. Khajetoorians - Institute for Molecules and Materials, Radboud University, 6525 AJ Nijmegen, The Netherlands

Complete contact information is available at:

https://pubs.acs.org/10.1021/acs.nanolett.1c00788

\section{Author Contributions}

T.C.H. and D.W. designed and T.C.H., B.K., and J.H.S. conducted the experiments. T.C.H., J.H.S., B.K., and D.W. analyzed the data. All authors contributed to discussions and writing of the manuscript and have given approval to the final version of the manuscript.

\section{Notes}

The authors declare no competing financial interest.

\section{ACKNOWLEDGMENTS}

This work is part of the research program of the Dutch Foundation for Fundamental Research on Matter (FOM, project no. 15PR3221), which is financially supported by The Netherlands Organization for Scientific Research (NWO). A.A.K. acknowledges the VIDI project "Manipulating the interplay between superconductivity and chiral magnetism at the single atom level" with project no. 680-47-534, which is financed by NWO.

\section{REFERENCES}

(1) Erbas-Cakmak, S.; Leigh, D. A.; McTernan, C. T.; Nussbaumer, A. L. Artificial Molecular Machines. Chem. Rev. 2015, 115, 10081. 
(2) Abendroth, J. M.; Bushuyev, O. S.; Weiss, P. S.; Barrett, C. J. Controlling Motion at the Nanoscale: Rise of the Molecular Machines. ACS Nano 2015, 9, 7746.

(3) Browne, W. R.; Feringa, B. L. Making molecular machines work. Nat. Nanotechnol. 2006, 1, 25.

(4) Saywell, A.; et al. Light-Induced Translation of Motorized Molecules on a Surface. ACS Nano 2016, 10, 10945.

(5) Ragazzon, G.; Baroncini, M.; Silvi, S.; Venturi, M.; Credi, A. Light-powered autonomous and directional molecular motion of a dissipative self-assembling system. Nat. Nanotechnol. 2015, 10, 70.

(6) Comstock, M. J.; et al. Reversible photomechanical switching of individual engineered molecules at a metallic surface. Phys. Rev. Lett. 2007, 99, 038301.

(7) Kudernac, T.; et al. Electrically driven directional motion of a four-wheeled molecule on a metal surface. Nature 2011, 479, 208.

(8) Alemani, M.; et al. Electric field-induced isomerization of azobenzene by STM. J. Am. Chem. Soc. 2006, 128, 14446.

(9) Grill, L.; et al. Rolling a single molecular wheel at the atomic scale. Nat. Nanotechnol. 2007, 2, 95.

(10) Choi, B. Y.; et al. Conformational molecular switch of the azobenzene molecule: A scanning tunneling microscopy study. Phys. Rev. Lett. 2006, 96, 156106.

(11) Ni, C.; Wang, J. Z. STM Studies on Molecular Rotors and Motors. Surf. Rev. Lett. 2018, 25, 1841004.

(12) Hla, S. W. Atom-by-atom assembly. Rep. Prog. Phys. 2014, 77, 056502 .

(13) Swart, I.; Sonnleitner, T.; Niedenführ, J.; Repp, J. Controlled Lateral Manipulation of Molecules on Insulating Films by STM. Nano Lett. 2012, 12, 1070.

(14) Simpson, G. J.; Garcia-Lopez, V.; Petermeier, P.; Grill, L.; Tour, J. M. How to build and race a fast nanocar. Nat. Nanotechnol. 2017, 12, 604 .

(15) Eigler, D. M.; Schweizer, E. K. Positioning Single Atoms with a Scanning Tunneling Microscope. Nature 1990, 344, 524.

(16) Bartels, L.; et al. Dynamics of electron-induced manipulation of individual $\mathrm{CO}$ molecules on $\mathrm{Cu}(111)$. Phys. Rev. Lett. 1998, 80, 2004.

(17) Komeda, T.; Kim, Y.; Kawai, M.; Persson, B. N. J.; Ueba, H. Lateral hopping of molecules induced by excitation of internal vibration mode. Science 2002, 295, 2055.

(18) Liljeroth, P.; Repp, J.; Meyer, G. Current-induced hydrogen tautomerization and conductance switching of naphthalocyanine molecules. Science 2007, 317, 1203.

(19) Baumberg, J. J.; Aizpurua, J.; Mikkelsen, M. H.; Smith, D. R. Extreme nanophotonics from ultrathin metallic gaps. Nat. Mater. 2019, 18, 668.

(20) Berndt, R.; et al. Photon-Emission at Molecular Resolution Induced by a Scanning Tunneling Microscope. Science 1993, 262, 1425.

(21) Berndt, R.; Gimzewski, J. K.; Johansson, P. Inelastic Tunneling Excitation of Tip-Induced Plasmon Modes on Noble-Metal Surfaces. Phys. Rev. Lett. 1991, 67, 3796.

(22) Neuman, T.; Esteban, R.; Casanova, D.; Garcia-Vidal, F. J.; Aizpurua, J. Coupling of Molecular Emitters and Plasmonic Cavities beyond the Point-Dipole Approximation. Nano Lett. 2018, 18, 2358.

(23) Rossel, F.; Pivetta, M.; Schneider, W. D. Luminescence experiments on supported molecules with the scanning tunneling microscope. Surf. Sci. Rep. 2010, 65, 129.

(24) Kazuma, E.; Jung, J.; Ueba, H.; Trenary, M.; Kim, Y. Real-space and real-time observation of a plasmon-induced chemical reaction of a single molecule. Science 2018, 360, 521.

(25) Zhang, L.; et al. Electrically driven single-photon emission from an isolated single molecule. Nat. Commun. 2017, 8, 580.

(26) Chen, G.; et al. Spin-Triplet-Mediated Up-Conversion and Crossover Behavior in Single-Molecule Electroluminescence. Phys. Rev. Lett. 2019, 122, 177401.

(27) Imada, H.; et al. Real-space investigation of energy transfer in heterogeneous molecular dimers. Nature 2016, 538, 364.
(28) Luo, Y.; et al. Electrically Driven Single-Photon Superradiance from Molecular Chains in a Plasmonic Nanocavity. Phys. Rev. Lett. 2019, 122, 233901.

(29) Zhang, Y.; et al. Visualizing coherent intermolecular dipoledipole coupling in real space. Nature 2016, 531, 623.

(30) Imada, H.; et al. Single-Molecule Investigation of Energy Dynamics in a Coupled Plasmon-Exciton System. Phys. Rev. Lett. 2017, 119, 013901.

(31) Zhang, Y.; et al. Sub-nanometre control of the coherent interaction between a single molecule and a plasmonic nanocavity. Nat. Commun. 2017, 8, 15225.

(32) Doppagne, B.; et al. Electrofluorochromism at the singlemolecule level. Science 2018, 361, 251.

(33) Rai, V.; et al. Boosting Light Emission from Single Hydrogen Phthalocyanine Molecules by Charging. Nano Lett. 2020, 20, 7600.

(34) Doppagne, B.; et al. Vibronic Spectroscopy with Submolecular Resolution from STM-Induced Electroluminescence. Phys. Rev. Lett. 2017, 118, 127401.

(35) Doppagne, B.; et al. Single-molecule tautomerization tracking through space- and time-resolved fluorescence spectroscopy. Nat. Nanotechnol. 2020, 15, 207.

(36) Doležal, J.; et al. Mechano-Optical Switching of a Single Molecule with Doublet Emission. ACS Nano 2020, 14, 8931.

(37) Doležal, J.; et al. Charge Carrier Injection Electroluminescence with CO-Functionalized Tips on Single Molecular Emitters. Nano Lett. 2019, 19, 8605.

(38) Kaiser, K.; Gross, L.; Schulz, F. A Single-Molecule Chemical Reaction Studied by High-Resolution Atomic Force Microscopy and Scanning Tunneling Microscopy Induced Light Emission. ACS Nano 2019, 13, 6947.

(39) Miwa, K.; Imada, H.; Kawahara, S.; Kim, Y. Effects of moleculeinsulator interaction on geometric property of a single phthalocyanine molecule adsorbed on an ultrathin $\mathrm{NaCl}$ film. Phys. Rev. B: Condens. Matter Mater. Phys. 2016, 93, 165419.

(40) Patera, L. L.; Queck, F.; Scheuerer, P.; Moll, N.; Repp, J. Accessing a Charged Intermediate State Involved in the Excitation of Single Molecules. Phys. Rev. Lett. 2019, 123, 016001.

(41) Schaffert, J.; et al. Imaging the dynamics of individually adsorbed molecules. Nat. Mater. 2013, 12, 223.

(42) Tao, M. L.; et al. Structural transitions in different monolayers of cobalt phthalocyanine film grown on $\mathrm{Bi}(111)$. J. Phys. D: Appl. Phys. 2016, 49, 015307.

(43) Peller, D.; et al. Sub-cycle atomic-scale forces coherently control a single-molecule switch. Nature 2020, 585, 58.

(44) Kröger, J.; Doppagne, B.; Scheurer, F.; Schull, G. Fano Description of Single-Hydrocarbon Fluorescence Excited by a Scanning Tunneling Microscope. Nano Lett. 2018, 18, 3407.

(45) Yang, B.; et al. Sub-nanometre resolution in single-molecule photoluminescence imaging. Nat. Photonics 2020, 14, 693.

(46) Repp, J.; Meyer, G.; Stojkovic, S. M.; Gourdon, A.; Joachim, C. Molecules on insulating films: Scanning-tunneling microscopy imaging of individual molecular orbitals. Phys. Rev. Lett. 2005, 94, 026803 .

(47) Lamb, W. E.; Retherford, R. C. Fine Structure of the Hydrogen Atom by a Microwave Method. Phys. Rev. 1947, 72, 241.

(48) Rybin, M. V.; Mingaleev, S. F.; Limonov, M. F.; Kivshar, Y. S. Purcell effect and Lamb shift as interference phenomena. Sci. Rep. 2016, 6, 20599.

(49) Zhang, F. L.; et al. Elucidating Molecule-Plasmon Interactions in Nanocavities with $2 \mathrm{~nm}$ Spatial Resolution and at the SingleMolecule Level. Angew. Chem., Int. Ed. 2019, 58, 12133.

(50) van Weerdenburg, W. M. J.; et al. A scanning tunneling microscope capable of electron spin resonance and pump-probe spectroscopy at $\mathrm{mK}$ temperature and in vector magnetic field. Rev. Sci. Instrum. 2021, 92, 033906.

(51) Kassem, S.; et al. Artificial molecular motors. Chem. Soc. Rev. 2017, 46, 2592. 
(52) Fang, Y. R.; Sun, M. T. Nanoplasmonic waveguides: towards applications in integrated nanophotonic circuits. Light: Sci. Appl. 2015, 4, No. e294. 